\title{
Extraction of 3D Geometrical Features of Biological Objects with 3D PCA Analysis and Applications of Results
}

\author{
Michal Rychlik and Witold Stankiewicz \\ Poznan University of Technology \\ Division of Machine Design Methods \\ Poland
}

\section{Introduction}

The Computer Aided Design (CAD) systems are very well known by designers in their every day practice and numerical analysis. Computer models of real objects with advanced numerical tools, significantly improves the quality and reduce time of design process. In addition to the three-dimensional modeling systems, there are many other tools and techniques (such as reverse engineering, rapid prototyping), which will further enhance the capabilities of engineers.

Many of those engineering technologies, especially CAD/CAM techniques, have an application not only in mechanical design. These tools also can be used in different disciplines, like biomechanics, bioengineering, biometrics, etc. This interdisciplinary area of knowledge takes advantage of Reverse Engineering, three-dimensional modeling and simulation, FEM 1 analysis and is equipped with Rapid Prototyping and CNC machines. The acquisition and processing of three-dimensional models with complicated shapes becomes the important issue in applications mentioned above. The 3D virtual models have numerous applications, simply such as visualization, but also more advanced like medical diagnostics (virtual endoscopes), pre-surgical planning (simulations of surgical operations), FEM and $\mathrm{CFD}^{2}$ analysis, CNC machining, Rapid Prototyping, preparation and fabrication of implants, etc. Several engineering technologies and tools can be used for advanced analysis of biological objects.

The first step in computer analysis of biological/medical objects is to obtain the correct and high accurate 3D model. This 3D modeling generally is made by reconstruction procedure. $3 \mathrm{D}$ reconstruction can be done by usage of medical imagining systems (such as CT, NMR) or 3D scanning systems. Reconstruction of 3D model based on $\mathrm{DICOM}^{3}$ images must be supported by image processing (segmentation of the region of interest) to extract 3D shape of the object. Dependent on the area of interest the bones, blood vessels or other soft tissues can be searched.

\footnotetext{
${ }^{1} \mathrm{FEM}$ - finite element method

${ }^{2} \mathrm{CFD}$ - computational fluid dynamics

${ }^{3} \mathrm{DICOM}$ - Digital Imaging in Communications in Medicine
} 
The main objective of this chapter is to present the possibility of a new use of "tools", known from engineering area, in the biomedical applications. One of the useful tools, known from technical applications (mainly in images and signal analysis) is Principal Component Analysis. This method is usually used for one or two dimensional "statistical" analysis, but three or more dimensions also can be analyzed. That multidimensional PCA analysis supported by other "high-tech" technologies (Reverse Engineering - 3D scanners, thermal camera, CT or NMR imagining), can generate very interesting results especially for 3D biomedical objects. In further sections the three applications (in anthropometrics, biometricsand for 3D geometry reconstruction procedure) of three-dimensional PCA analysis of biological objects will be presented and discussed.

\section{Methods of modal analysis}

In this chapter authors present modal analysis methods which can be used for geometry description of three dimensional objects. This methods are used for simplify and minimize the number of parameters which describe 3D objects.

The kinds of modal method (mathematical, physical or empirical) which are applied to analysis have a fundamental importance onto results. One of the method which based on modal decomposition is PCA (Principal Component Analysis, known also as POD - Proper Orthogonal Decomposition). If empirical modes (PCA) are optimal from viewpoint of information included inside of the each modes [Holmes, Lumley and Berkooz, 1998], also others decompositions based on mathematical (e.g. spherical harmonics) or physical modes (vibration modes) are also used.

The goal of using mathematical modes is conversion physical features onto mathematical features (synthetic form). In the event of the mathematical modes usually the features which describing geometry of $3 \mathrm{D}$ object is save as the vectors. Each vector is obtained through splitting of the 3D model on the several classes (different diameter spheres) and calculates common areas for $3 \mathrm{D}$ object and surface of individual spheres. All areas are described by set of vectors (spherical functions). For spherical functions Fourier transformation is used. After this operation the new unit is inserting. Goal of unit is made easier multidimensional description of features vectors. For representation of features vectors spherical harmonics are used (Fig. 1.).

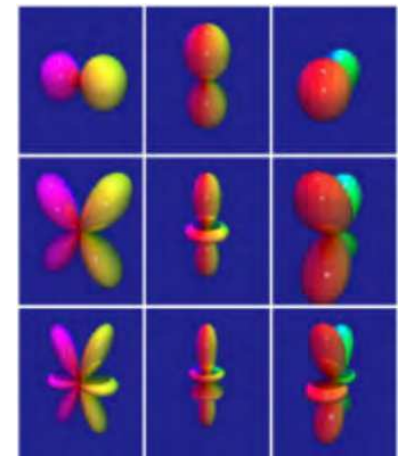

(a)

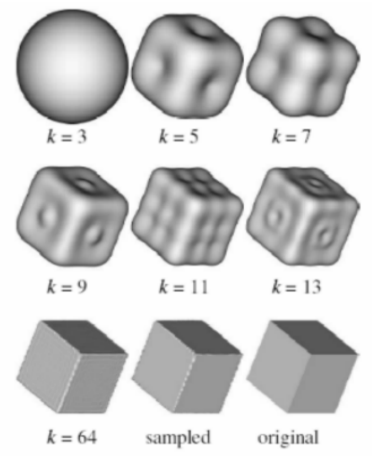

(b)

Fig. 1. Example of spherical harmonics of 3D model of plane (a) and application spherical harmonic to reconstruction of geometry of the cube (b) [Vranic and Saupe, 2002] 
Application of spherical modes is not "optimal" solution and sometimes cause of increasing complication of the computation because all objects are approximated by deformed sphere. Reconstruction of cube geometry can be done by very many numbers of spherical harmonics. This problem is analogous to Fourier decomposition of rectangular signal.

The second group of modal decomposition of 3D objects is present by physical modes (mechanical modes). This modes - known also as the vibration modes - are obtained by solution of equitation of own issue for resilience model of analyzing object. Vibration modal decomposition provides alternative parameterization of degree of freedom of the structure (only translation of the nodes in $\mathrm{x}, \mathrm{y}, \mathrm{z}$ directions) based on proper vibration of the objects and correlated frequencies. The most often are used low frequencies of the modes which described vectors of deformations for individual nodes of FEM grid. This way is possible the deformation of geometry of basic object and to fit it into searched object. Vibration modes compute for rigid body are responsible for translations and rotations of 3D model. Vibration modes compute for spring body describe different variation of the shape of basic model (Fig. 2.).
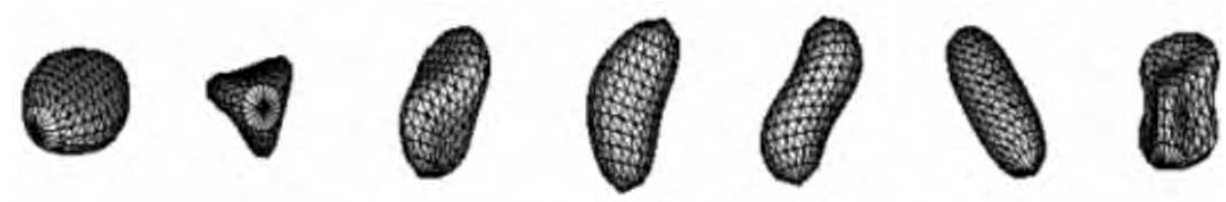

Fig. 2. Graphical representation of seven low frequencies vibration modes for surface model of ellipsoids [Syn and Prager, 1994]

PCA transformation gives orthogonal directions of principal variation of input data. Principal component which are connected with the largest eigenvalue, represent factor (direction) of the largest variation of data in data space. Variation is described by eigenvalue connected with the first principal component. The second principal component describes the next in order, orthogonal direction in the space with the next largest variation of data. Usually only few first principal components are responsible for a majority variations of the data. Data projected onto other principal components often have small amplitude and don't cross a amplitude of measurement noise. Therefore they can be deleted, without dangerous of decreasing of the accuracy.

\section{Principal Component Analysis (empirical modes)}

For reconstruction of the 3D geometry, "low-dimensional" decomposition based on Principal Component Analysis (PCA) can be used [Benameur S., Mignote M., Parent S., Labelle H., Skalli W., De Gusie J., 2001]. PCA provides a "relevant" set of basis functions, which allow identification of a low-dimensional subspace [Holmes, Lumley and Berkooz, 1996].

The used algorithm is based on statistical representation of the random variables.

"The prefix eigen- is adopted from the German word "eigen" for "own" in the sense of a characteristic description 
The shape of the each object is represented in the data base as the set of 3D point clouds. Each point clouds is described by a vector (1):

$$
S_{i}=\left[s_{i 1}, s_{i 2}, \ldots, s_{i N}\right]^{T}, i=1,2, \ldots, M,
$$

where $s_{i j}=(x, y, z)$ describes coordinates of the points in Cartesian system, $M$ is the number of the objects which are in database, $N$ is the number of the points in single point cloud. In the next step the mean shape $\bar{S}$ and covariance matrix $C$ are computed (2):

$$
\bar{S}=\frac{1}{M} \sum_{i=1}^{M} S_{i}, C=\frac{1}{M} \sum_{i=1}^{M} \tilde{S}_{i} \tilde{S}_{i}^{T},
$$

The difference between mean and object that is in data base are describe by the deformation vector $\tilde{S}_{i}=S_{i}-\bar{S}$. The statistical analysis of the deformation vectors gives us the information about the empirical modes. Modes represent the geometrical features (shape) but also can carry other information like texture, map of temperature and others. Only few first modes contains most information, therefore each original object $S_{i}$ is reconstructed by using some K principal components (3):

$$
S_{i}=\bar{S}+\sum_{k=1}^{K} a_{k i} \Psi_{k}, i=1,2, \ldots, M,
$$

where $\Psi_{k}$ is an eigenvector representing the orthogonal mode (the feature computed from data base), $a_{k i}$ is coefficient of eigenvector.

Energy (equivalent of quantity of information) transmitted by eigenvector $m$ is appointed on following equation:

$$
E=\frac{\Psi_{m}}{\sum_{k=1}^{M} \Psi_{k}}
$$

The example of the low dimensional reconstruction for three different values of the coefficients is presented on the Figure 3. Every manipulations of coefficients value of the modes, changing geometry of the objects.

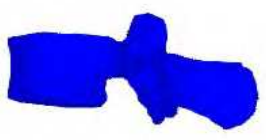

$a_{k i}=0$

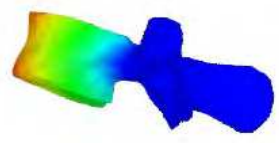

$a_{k i}=-5 \cdot 10^{-6}$

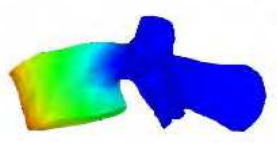

$a_{k i}=5 \cdot 10^{-6}$

Fig. 3. The visualization ofthe low dimensional reconstruction for L4 vertebra: average value (left side) and two different deformation which are compute for different values of the coefficients (middle and right side). 


\section{Applications of Principal Component Analysis of 3D biological objects}

Several engineering technologies can be used for advanced analysis of biological objects. In further chapters the three applications, anthropometric (femur bones), biometric (human faces), geometry reconstruction (lumbar vertebra) of three-dimensional models will be presented and discussed. For biometric database the thermal (infrared) images was tested as support information.

\subsection{Biometric application of 3D PCA}

The security and access systems are very important and rapidly advancing, not only in computer vision. Such systems are used obviously during passenger control on the airport or boundary crossing [Schneider W., 2007]. Biometrics identify people by measuring some aspects of individual anatomy or physiology - such as hand geometry or fingerprint, some deeply ingrained skill, or other behavioral characteristic - handwritten signature, or something that is a combination of the two - voice [Anderson, R. J., 2008]. In generally the biometrics can be sorted into two types [Mainguet J-F. , 2004]:

a. physical - face, fingerprint, hand/finger, iris, ear, retinal, DNA, vein, blood pulse, dental, lips, nail;

b. behavioral - voice, gait, tapping, signature, keystroke dynamics, mouse dynamics.

The face recognition method is the oldest and the most "natural" method of identification person's. Recognizing people by their facial features is going back at least to our early primate ancestors.

The disadvantage of the most commonly used recognition techniques is their insufficient reliability. A 2D dimensional photo cannot be measured like a landscape and simply doesn't contain the same amount of information as the 3D "photo" [Xiaoguang Lu, 2006]. This problem is especially essential for twins, when the similarity of face shape is very high [Anil K. J., at al., 2002].

Facial identification reads the peaks and valleys of facial features. These peaks and valleys are known as nodal points ( 80 nodal points exist in a human face, but usually only 15-20 are used for identification - known as "Golden triangle" region between the temples and the lips.

\subsubsection{Materials - Acquisition of input data}

For obtain the 3D input data the 3D scanning system (the structural light scanner) was used. The two groups of biological models were measured: set of human faces and set of femur bones. Each input object was scanned and 3D surface model was computed.

To increase the accuracy of PCA analysis, each face was described (Fig. 4.) by individual points cloud (40k points) instead of few markers from "Golden triangle" area.

Database for biometric PCA analysis is prepared onto the multiple human faces. There are 39 faces (Fig. 5.) with neutral expression of different persons. To improve sensitivity of presented method, three sets (6 faces) of the twin's faces in the database were included. There are two sets of identical - monozygotic twins and one set of fraternal - dizygotic twins. 

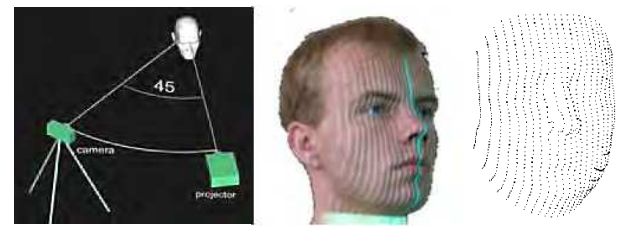

Fig. 4. Data acquisition process (from left): hardware configuration, captured image, input data set (points cloud).

Second part of input data for biometric database is set of two-dimensional infrared images (thermal images). In this research the thermal images of 17 different persons were collected (Fig. 5.). For acquisition of infrared images the thermal camera was used. All pictures were collected in the same conditions of the measurement: room $\left(+22^{\circ} \mathrm{C}\right)$ and camera settings: matrix VOx $320 \times 240$ pixels, thermal resolution $+/ 0.03^{\circ} \mathrm{C}$, range of measured temperature: $26-400^{\circ} \mathrm{C}$.

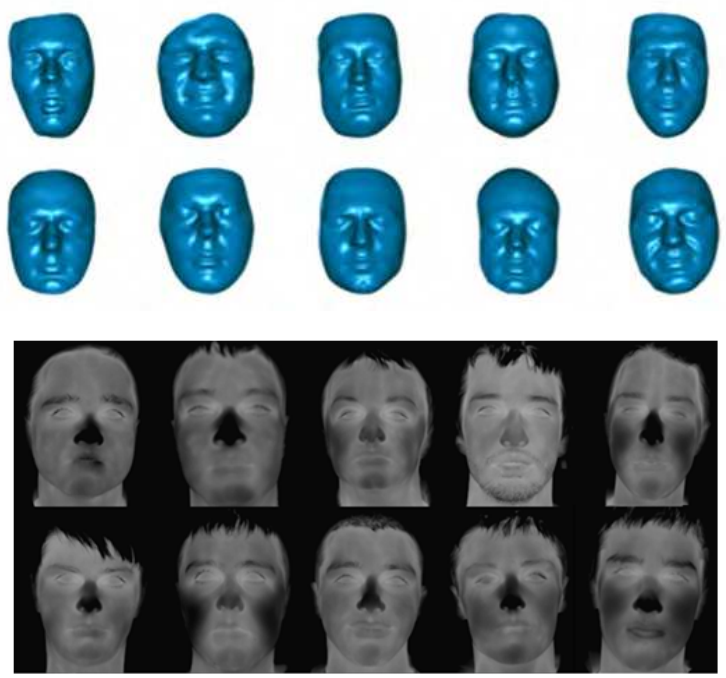

Fig. 5. An example of the models in database (from the left): 3D faces, 2D thermal images.

\subsubsection{Data registration}

The Principal Component Analysis requires the same position, orientation and topology of the data input (the same number of nodes, matrix connection, etc.) for all objects. To achieve this, each new object added to database must be registered.

For face the origin point $(0,0,0)$ of coordinate system is arranged in cross section of two lines: vertical middle line on the face, horizontal - eyes line. The registration is made in two steps. First step (preliminary registration) is the rigid registration. It consists of simple, affine geometrical transformation of object in three-dimensional space (rotation and transformation). For control of this process three points are used: two points in the centers of the eyes, one on the top of the nose (Fig.6.). 


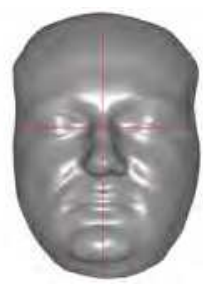

(a)

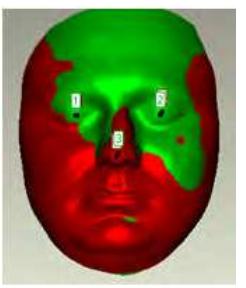

(b)

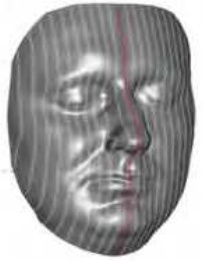

(c)

Fig. 6. Data registration: a) origin point, b) three point of rigid registration, c) 3D curves network.

The second step is connected with curves extraction. Independently from face size, always 201 curves on face are only. To achieve this, scaling of space between curves is used. For infrared images only the first step of registration was done.

\subsubsection{Results of 3D PCA analysis - Face print}

For prepared database of human faces (39 faces of young persons) the PCA analysis was performed. The result of this operation is the mean object, thirty nine modes (Fig. 7) and coefficients (Tab. 1.).

The first thirty eight modes include one hundred percent of information about decomposed geometry. Mode thirty nine contains only a numerical noise. For further reconstruction of face geometry only first twenty five modes are used.

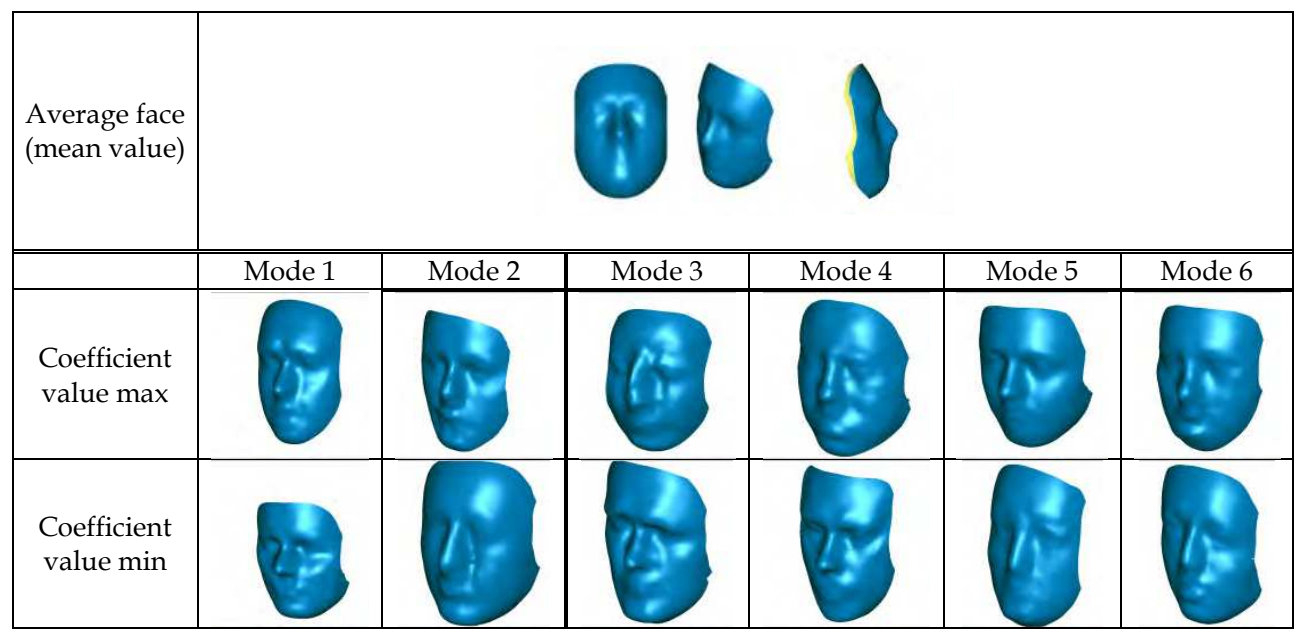

Fig. 7. Visualization of the average value and first nine empirical modes of faces (for maximum and minimum values of coefficient). 


\begin{tabular}{ccc}
\hline $\begin{array}{c}\text { Number } \\
\text { of the mode }\end{array}$ & $\begin{array}{c}\text { Participation } \\
\text { of the mode [\%] }\end{array}$ & $\begin{array}{c}\text { Total participation } \\
\text { of the modes [\%] }\end{array}$ \\
\hline 1 & 57,2010066 & 57,2010066 \\
2 & 13,4976482 & 70,6986548 \\
3 & 5,9128812 & 76,6115360 \\
4 & 4,3703630 & 80,9818990 \\
5 & 4,0161309 & 84,9980299 \\
6 & 3,1945602 & 88,1925901 \\
7 & 2,6277538 & 90,8203439 \\
8 & 1,3584618 & 92,1788057 \\
9 & 1,2914768 & 93,4702825 \\
10 & 0,9734244 & 94,4437069 \\
11 & 0,8401794 & 95,2838863 \\
12 & 0,6097119 & 95,8935982 \\
13 & 0,4819451 & 96,3755433 \\
14 & 0,4433567 & 96,8189000 \\
15 & 0,3511382 & 97,1700382 \\
16 & 0,3001483 & 97,4701865 \\
17 & 0,2681638 & 97,7383503 \\
18 & 0,2577919 & 97,9961422 \\
19 & 0,2179374 & 98,2140797 \\
20 & 0,1973631 & 98,4114428 \\
21 & 0,1767063 & 98,5881491 \\
22 & 0,1608646 & 98,7490137 \\
23 & 0,1512224 & 98,9002361 \\
24 & 0,1311154 & 99,0313516 \\
25 & 0,1250506 & 99,1564022 \\
\hline
\end{tabular}

Table 1. Participation of the modes in faces geometry reconstruction.

Modes describe the features of the faces. Two first modes characterize global transformation: first mode changing of the face size in vertical direction, second mode changing the size in horizontal direction. Further modes describe more complex, local deformations. For example third mode is responsible for changes in nose and eyes areas. Results of the statistical analysis (empirical modes) can be used for reconstruction of geometry (in CAD systems) of individual features of the object. Those 3D models of faces can be used in many applications: 3D visualizations, Rapid Prototyping technologies, surgical planning, archivisation and many others.

Each face in database has unique set of coefficient values "faceprint" (Fig. 8.) - individual ID code like fingerprints. As the authorization key the set of coefficient values for the faces can be used. Each key describes individual shape of face and can be decoded and compared with the original data of user to obtain access to restricted area or data files. 


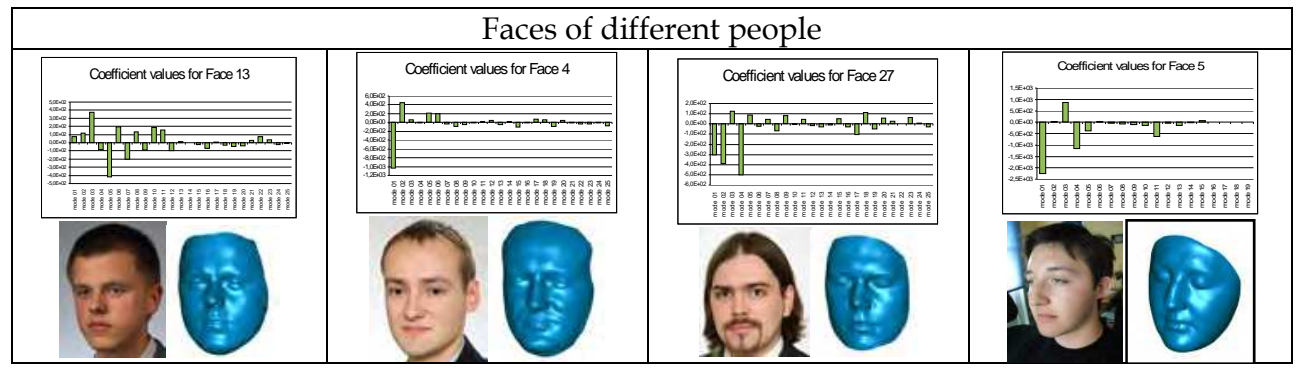

Fig. 8. Faceprints (ID codes) for few faces from database (for each object is presented - graph of coefficient values, original photo and 3D face model).

\subsubsection{Results of 2D PCA analysis - Thermal images}

As a support for three-dimensional face recognition, additional information - the thermal images - can be used [Akhloufi, M, Bendada A., 2008, Prokoski. F., 2000]. The twodimensional infrared images can be added to database as a "fourth" dimension. For prepared infrared images of human faces the 2D PCA analysis was done. The result of this operation is the mean face (Fig. 9.), seventeen modes and set of coefficients.

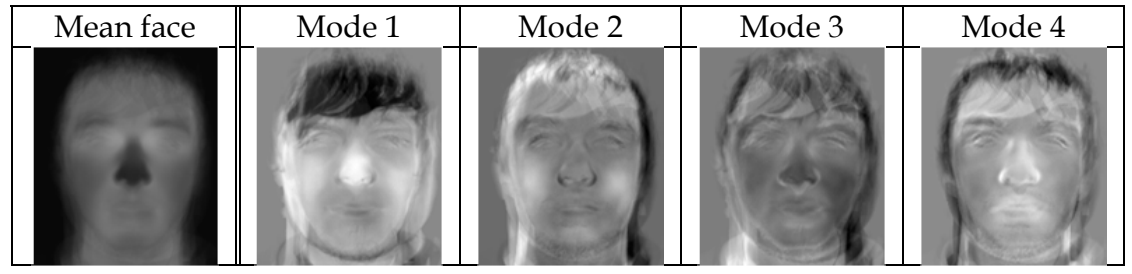

Fig. 9. Visualization of the mean face and first four empirical modes of infrared images.

For presented analysis the sixteen modes include one hundred percent of information about decomposed thermal images (Table 2.). Seventeenth mode contains only a numerical noise.

\begin{tabular}{ccc}
\hline $\begin{array}{c}\text { Number } \\
\text { of the mode }\end{array}$ & $\begin{array}{c}\text { Participation } \\
\text { of the mode [\%] }\end{array}$ & $\begin{array}{c}\text { Total participation } \\
\text { of the modes [\%] }\end{array}$ \\
\hline 1 & 26.4191478 & 26.4191478 \\
2 & 16.7302890 & 43.1494367 \\
3 & 10.9104597 & 54.0598965 \\
4 & 8.3397586 & 62.3996550 \\
5 & 6.5525733 & 68.9522283 \\
6 & 5.6298384 & 74.5820668 \\
7 & 4.4142731 & 78.9963398 \\
8 & 3.6441677 & 82.6405075 \\
9 & 3.3063140 & 85.9468215 \\
10 & 2.7908036 & 88.7376251 \\
\hline
\end{tabular}

Table 2. Participation of the first 10 modes PCA decomposition of thermal images. 
Similarly to 3D data, each infrared image of the face in database has unique set of coefficient values - "thermal faceprints" (Fig. 10.). This additional information can be associated with 3D data to increase the level of security and can complicate the trials of fake the system.

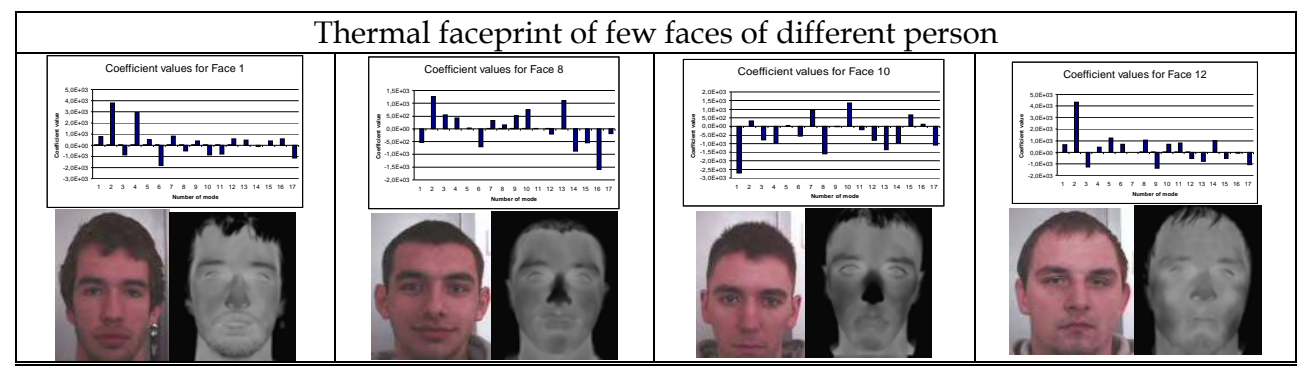

Fig. 10. Thermal faceprint for few faces from infrared database (for each object is presented graph of coefficient values, original photo and thermal image).

\subsubsection{Analysis of similarity and differences of twin's faces}

For testing of sensitivity level of presented faceprint method the twin's faces was used. For numerical experiment the database with 19 faces (which include three couples of twins - two couples of monozygotic twins and one couple of dizygotic twins) was prepared.

For better comparison and computation of similarity of twin's faces the additional analysis was done. PCA analysis give information about common shape and differences between each face of twins couple. Results of this analysis (surface map of differences are presented) is presented for one monozigotic and one dizygotic twins (Fig. 11., Fig. 12.).

Faces of monozygotic twins

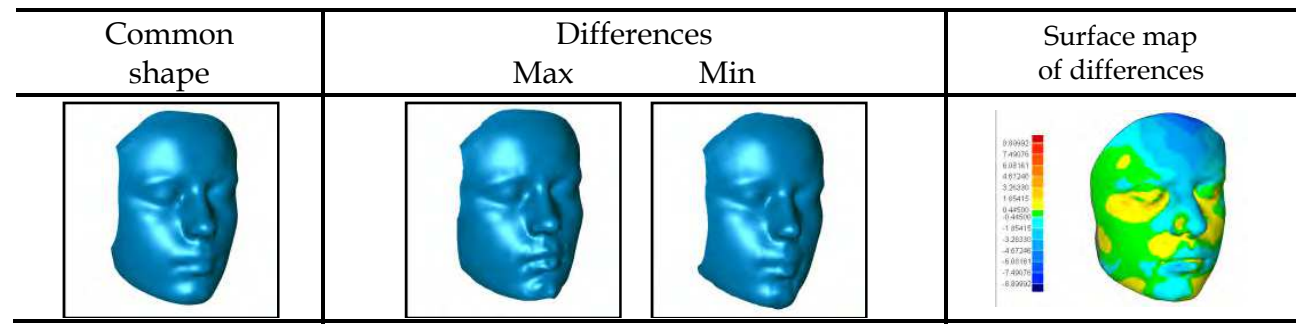

Fig. 11. Visualizations of the common shape (average face) and differences (empirical mode and surface map of difference) for monozygotic twins (gender: female). 
Faces of dizygotic twins

\begin{tabular}{c|c|c|c}
\hline $\begin{array}{c}\text { Common } \\
\text { shape }\end{array}$ & \multicolumn{2}{|c|}{ Differences } & \multicolumn{2}{c}{$\begin{array}{c}\text { Surface map of } \\
\text { differences }\end{array}$} \\
\hline & & & \\
\hline
\end{tabular}

Fig. 12. Visualizations of the common shape (mean face) and differences (empirical mode and surface map of difference) for dizygotic twins (gender: male).

For comparison of similarity of twins faces the value of surface deviation was done. For faces of monozygotic couple the value of standard deviation was $1,19 \mathrm{~mm}$ and average distance $0,29 \mathrm{~mm}$. For faces of dizygotic twins standard deviation is near $1,79 \mathrm{~mm}$ and average distance 0,83 . Each twin's face in database has unique set of coefficient values - "ID code" (Fig. 13.).

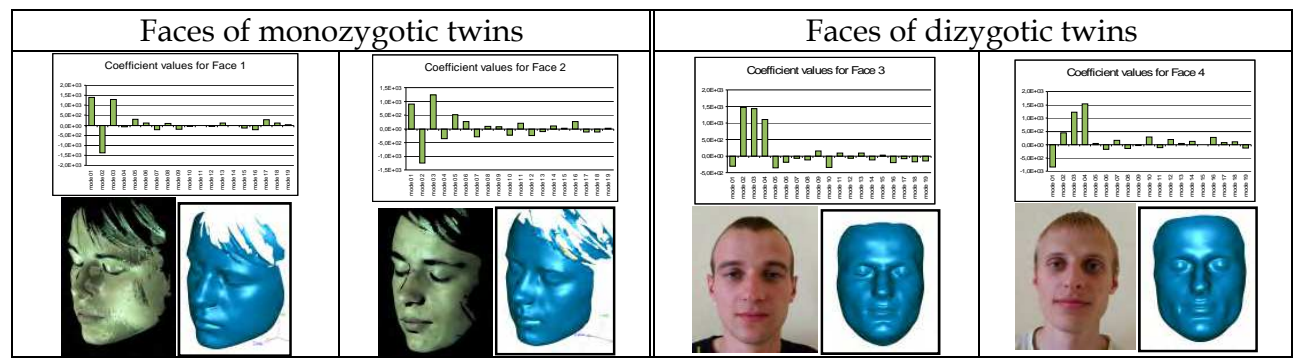

Fig. 13. Face ID code for faces of two types of twins (from upper left): face code - coefficient values, original photo, 3D face mode.

Also for identical (for human eye view) monozygotic twins this "face-code" is incomparable for each one face similar like for fingerprints (Anil K., 2002 ).

Trend curves (Fig. 14) of coefficients values showing how faces of twins are similar between each other. In general shape, curves (trends) are the same but in local values they have small differences. By this way the description ofsimilarity level between faces of the same couple of twins. As much faces are different from each other, than curves are automatically more far from each other.

When we try comparing two trends curves from different couple of twins, we can observe very big difference. Each face have own unique trend curve even for twins. 


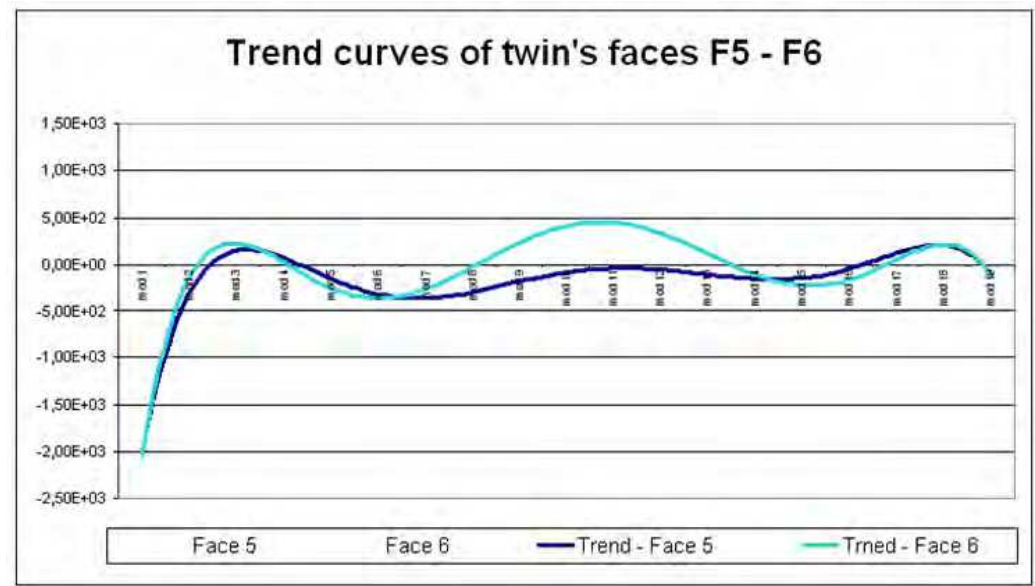

(a)

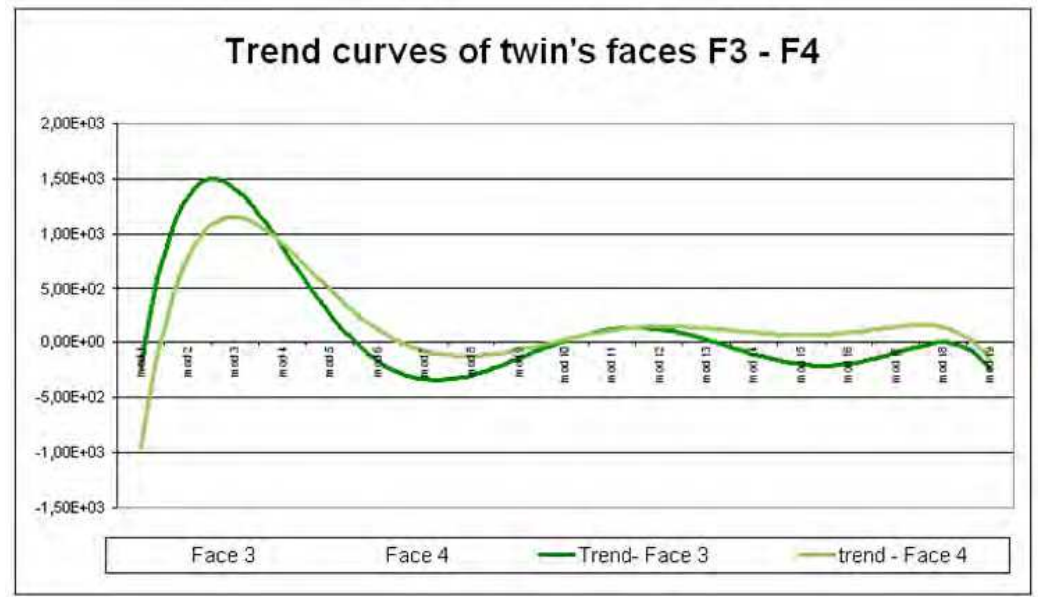

(b)

Fig. 14. Comparison of trend curves of coefficient values of modes: a) monozygotic twins, b) dizygotic twins.

\subsubsection{Results of 3D PCA analysis - Hand shape biometrics}

For identity of persons as a input data the many different information can be used. One of them is the hand shape. Hand geometry verification systems use geometric measurements of hand as the features for the verification of individuals. Usually they measure and analyze the overall structure, 2D shape (silhouette) and proportions of the hand e.g. length, width and thickness of hand, fingers and joints [Sanchez-Reillo R. at al., 2000]. Hand geometry biometrics systems measure up to 90 parameters (Fig. 15). Hand biometrics systems have some limitations, especially for people with severe arthristis who cannot spread their hands on the special hand reader. 

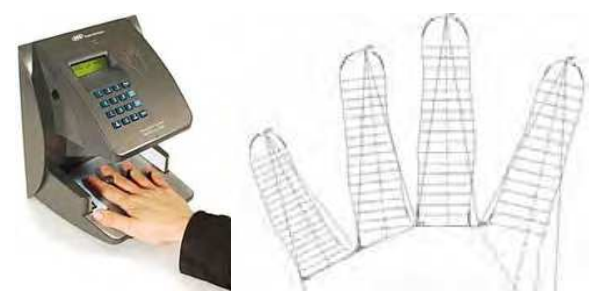

Fig. 15. Hand biometrics (from left): 2D biometric hand reader, segmentation of length and weight of the hand fingers features [Sanchez-Reillo R. at al., 2000].

Basis on faceprint methodology (described in chapter 4.1.3.) the 3D hand biometric identification system was prepared and tested.

The data input for numerical experiment was obtained by using the 3D optical scanner analogues to obtain the geometry of the bones. Measurements of hand geometry are easy to collect but for obtain full 3D information the special platform with mirrors was created. The system of mirrors and settings for localization of fingers (Fig. 16.) are comparable like used in regular 2D silhouette scanners [Jain A.K., at al., 1999].

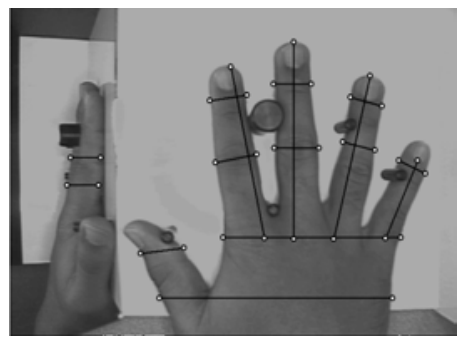

Fig. 16. Hand positioning system with mirror [Jain A.K., at al., 1999]

For test the database contains of 10 different 3D models of human hand (Fig. 17.). For each hand the registration procedure was done. As final result the set of polygon meshes $(6,5 \mathrm{k}$ triangles) describing hands was obtained and used in further 3D PCA analysis.
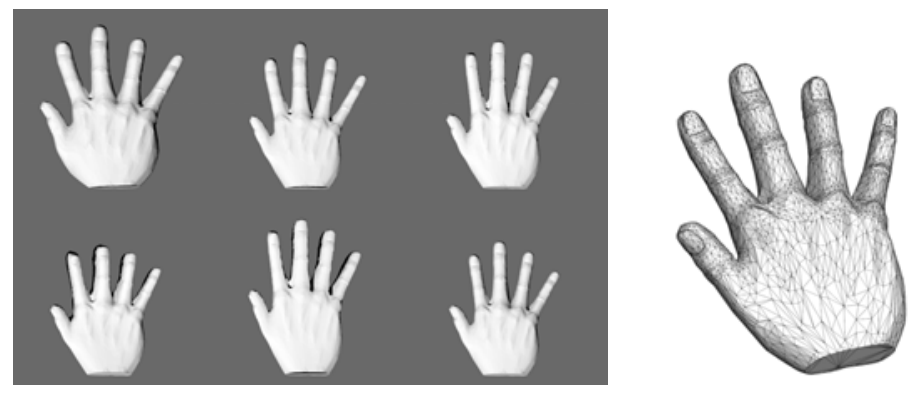

Fig. 17. Input data for 3D hand PCA analysis: five 3D models of different hands, example of mesh grid. 
For this analysis the nine modes include one hundred percent of information about hand geometry (Table 3.). The last, tenth mode, contain only a numerical noise.

\begin{tabular}{ccc}
\hline $\begin{array}{c}\text { Number of } \\
\text { the mode }\end{array}$ & $\begin{array}{c}\text { Participation } \\
\text { of the mode [\%] }\end{array}$ & $\begin{array}{c}\text { Total participation } \\
\text { of the modes [\%] }\end{array}$ \\
\hline 1 & 94.2193186 & 94.2193186 \\
2 & 1.9215473 & 96.1408659 \\
3 & 1.4766160 & 97.6174819 \\
4 & 0.8838991 & 98.5013809 \\
5 & 0.8546490 & 99.3560300 \\
6 & 0.3615261 & 99.7175561 \\
7 & 0.1225032 & 99.8400593 \\
8 & 0.0912159 & 99.9312752 \\
9 & 0.0687248 & 100.0000000 \\
10 & 0.0000000 & 100.0000000 \\
\hline
\end{tabular}

Table 3. Participation of the first 10 modes PCA decomposition of handgeometry.

Comparable like it was for 3D faces, the same situation is for hands. Individual modes characterize principal features of hand: size of hand, length of fingers, thickness of fingers and shape of hand regions (Fig. 18).

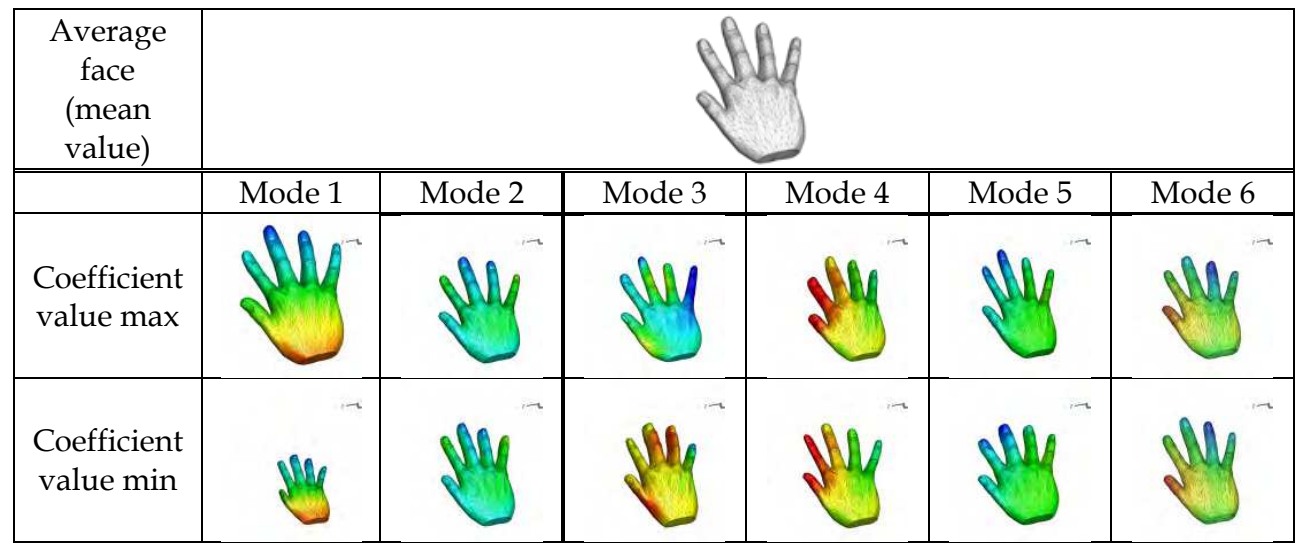

Fig. 18. Visualization of the average value and first nine empirical modes of faces (for maximum and minimum values of coefficient).

Each hand in database has unique set of coefficient values "handprint" (Fig. 19.) individual ID code like fingerprints just based on full 3D hand geometry. As the authorization key the set of coefficient values for the hands can be used. Each key describes individual 3D geometry of hand and can be decoded and compared with the original data of user to obtain access to restricted area or data files. 


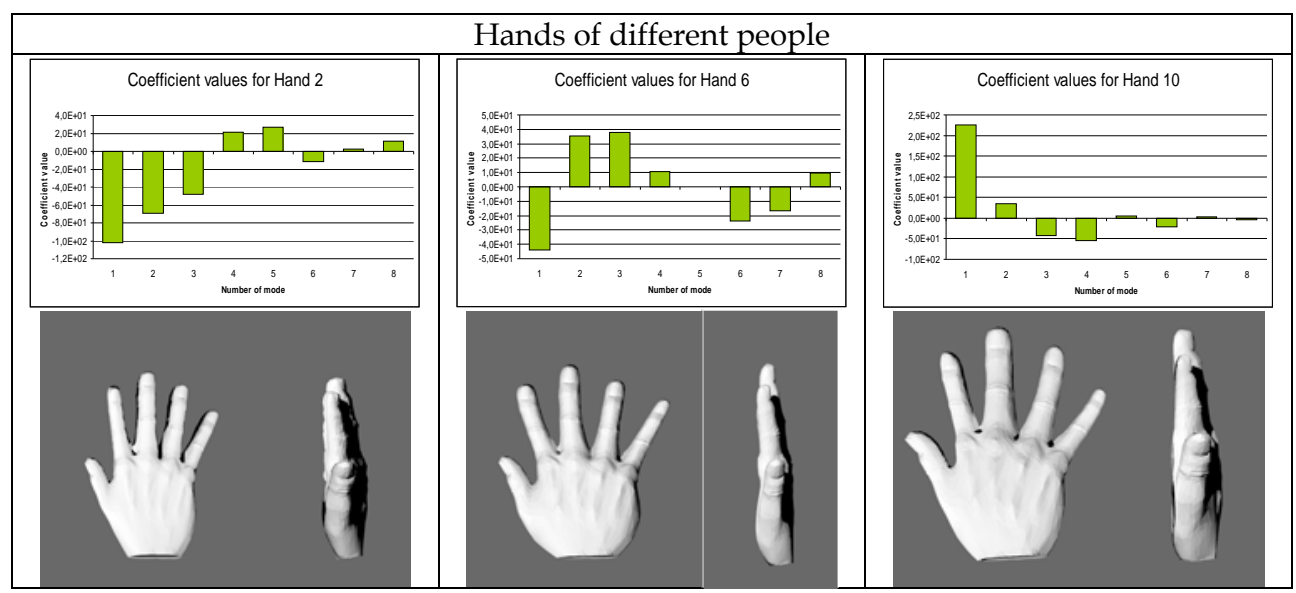

Fig. 19. Handprints (ID codes) for few hands from database (for each object is presented graph of coefficient values, two views of 3D hand model).

\subsection{Anthropometric application of 3D PCA}

Anthropometry is the science of measuring the human body (e.g. height, weight) and size of component parts, including skinfold thickness, to study and compare the relative proportions. Measurements can be done under normal and abnormal conditions. The results of measurements done for group or population of people are organised in special databases. The basic problem which exists in most of databases is not enough information. Most of them are old and not includes all interesting dimensions. Traditional anthropometric database contains information only about some characteristic points (other parameters are not described). The set of the bones are described only in two dimensional space [Jantz, R.L. and P.H. Moore-Jansen, 1988], by the collection of linear and angular dimensions (Fig.20.).

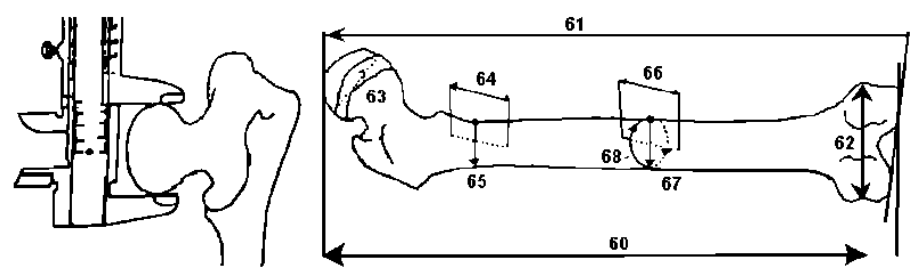

Fig. 20. Example of measurement process and view on a few characteristic points for femur bone [Moore-Jansen at al., 1994]

Three dimensional knowledge about mean geometry of the bones are not exist. Usually data acquisition process is prepared with usage of the conventional osteological instruments (measurements equipment like: caliper, spreading calliper, or osteometric board - Fig.21.). 


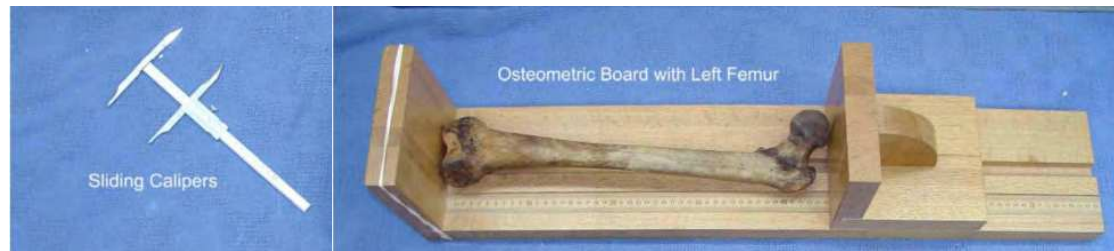

Fig. 21. Osteological instruments usually used for measuring the femur bone [Vitek C., 2005]

The solution of presented problem is proposition to use the Reverse Engineering techniques for measurement process (to achieve precision 3D data) and modal analysis (PCA) to compute the 3D anthropometric database. These aspects are presented further in details.

\subsubsection{Acquisition of input data}

For creation of database, the set of real bones was used. In this work was used the 15 femur bones (Fig. 22) - 6 female, 9 male - which are obtained from Poznan University of Medical Science.

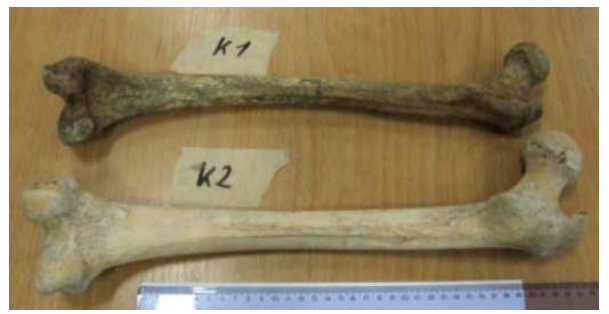

Fig. 22. View of two different female femur bones.

For acquisition of data the 3D structural light scanner was used (Fig. 23.). To acquire full 3D geometry, each bone was measured from 15 different directions with accuracy $0,05 \mathrm{~mm}$. Effect of measure process, is set of 3D points clouds (Fig. 23). All points clouds must be registered (orientation and connection between clouds).

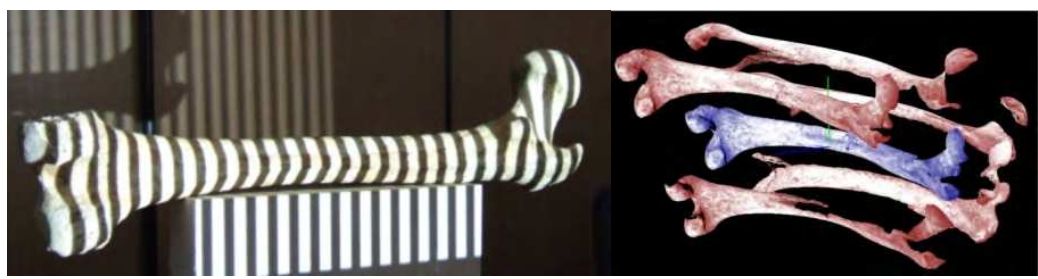

Fig. 23. Data acquisition process on 3D structural light scanner and set of points clouds.

The result of registration is one points cloud which consist about 1,5 mln points (Fig. 24). As the final step is generated 3D triangle surface grid. 


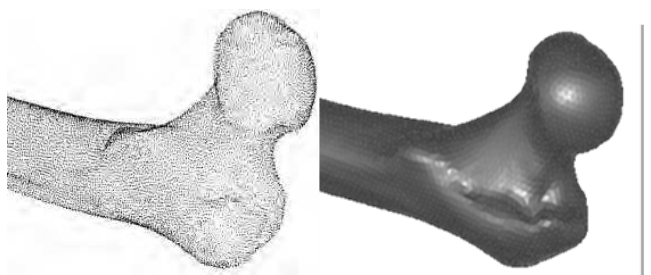

Fig. 24. Complete points cloud (1,5 mln points) and final triangle surface grid.

\subsubsection{Data registration}

The registration procedure forbones was made also in two steps. First step (preliminary registration) is simply rigid registration. The second step is the viscous fluid registration. For this registration the modified Navier-Stokes equation in penalty function formulation (existing numerical code [Morzynski M., at al., 1999]; source segment [Bro-Nielsen, M., Gramkow, C., 1996]) is used (5):

$$
\underbrace{\dot{V}_{i}+V_{i, j} V_{j}-\frac{1}{\operatorname{Re}} V_{i, j j}+\frac{\varepsilon-\lambda}{\rho} V_{j, j i}}_{\text {existing numerical code }}+\underbrace{(f-g) f_{, i}}_{\text {source segment }}=0
$$

where $\rho$ - is fluid density, $V_{i}$ - velocity component, Re - Reynolds number, $\lambda$ - bulk viscosity. In this application, parameters $\varepsilon$ and $\lambda$ are used to control the fluid compressibility, $f$ is the base object and $g$ is the target object (input model). The object is described by the grid nodes (FEM grid). The displacements of the nodes are computed from integration of the velocity field.

Every based and new model is represented by several cross-sections with the same transfer area (Fig. 25.). The transfer area is needed for topology conservation.
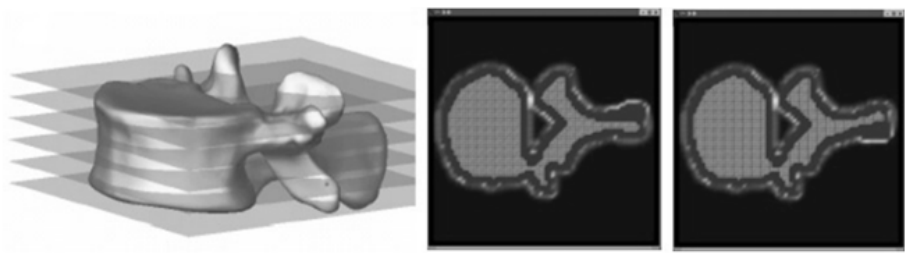

Fig. 25. Cross sections of the vertebra - orientation of the planes and two examples of 2D slices (from left): based vertebra geometry, new geometry of input vertebra (dark colour around bone- the transfer area).

Source term is calculated from the difference of the two images in grey scale (6):

$$
F_{i}=-(f-g) f_{, i}
$$

where $f$ and $g$ are areas in grayscale $f$ - base image (base vertebra), $g$ - target image (input vertebra). The parameters of the flow are the same as for the compressible fluid with very viscosity. 
Computed flow field provides information about translations of the nodes in both sections. After computation we obtain dislocation of nodes of the base grid onto new geometry (Fig. 26.).

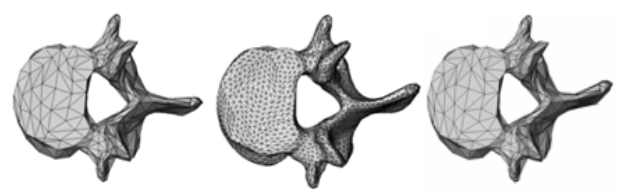

Fig. 26. Grid deformation (from the left): base object (base grid), new object inserted to database, base grid on geometry of the new objects.

\subsubsection{Results of 3D PCA analysis - Femur bones}

The database used in analysis contains 15 femur bones. Each bone has different geometry and is described by triangle surface grid (Fig. 27) with the same structure (14 thousands nodes, 30 thousands elements).

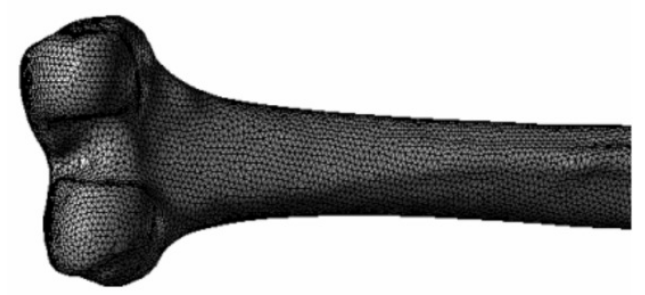

Fig. 27. Triangle surface grid of femur bone.

For this database the Principal Component Analysis was done. The result of this operation is the mean object, fifteen modes and coefficients.

The first fourteen modes include $100 \%$ of information about decomposed geometry (Table 4). Mode fifteen contain only a numerical noise and they are not used for further calculations.

Modes describing the features of the femur bones (Fig. 28). The first mode describes transformation of the length of the femur bone. Second mode represent position conversion of the head of the bone, third describe change the arc of the shaft (body). Next modes describing more complex deformations, e.g. fourth mod describe change the position of the greater trochanter and lesser trochanter, also thickness of the shaft (body).

In this experiment the value of average error for reconstructed geometry of the bone was equal $0,3 \mathrm{~mm}$ (reconstruction done with using of 14 modes).

Study of the value of the coefficients, give us additional information about the analyzed bones. For presented database we can found, correlation between coefficient value of first mode and gender. Negative coefficient value "_" of first mode is connected with female bones (one exception bone $\mathrm{nr} 8$ ), when positive coefficient value " + “ describe male bones (Fig. 29.). 


\begin{tabular}{ccc}
\hline $\begin{array}{c}\text { Number of } \\
\text { the mode }\end{array}$ & $\begin{array}{c}\text { Participation of the } \\
\text { mode [\%] }\end{array}$ & $\begin{array}{c}\text { Total participation of } \\
\text { the modes [\%] }\end{array}$ \\
\hline 1 & 74.9212416 & 74.9212416 \\
2 & 10.5438352 & 85.4650767 \\
3 & 4.2699519 & 89.7350286 \\
4 & 3.3128685 & 93.0478971 \\
5 & 1.6659793 & 94.7138765 \\
6 & 1.4234329 & 96.1373093 \\
7 & 1.0359034 & 97.1732127 \\
8 & 0.6781645 & 97.8513772 \\
9 & 0.5866122 & 98.4379894 \\
10 & 0.4796167 & 98.9176061 \\
11 & 0.3301463 & 99.2477523 \\
12 & 0.3080968 & 99.5558492 \\
13 & 0.2516839 & 99.8075330 \\
14 & 0.1924670 & 100.0000000 \\
15 & 0.0000000 & 100.0000000 \\
\hline
\end{tabular}

Table 4. Participation of the modes in decomposition.

\begin{tabular}{|c|c|c|c|c|}
\hline Mean value & & & -2 & \\
\hline $\begin{array}{l}\text { Number of the } \\
\text { mode }\end{array}$ & Mod 1 & Mod 2 & Mod 3 & Mod 4 \\
\hline $\begin{array}{l}\text { Min value of } \\
\text { the coefficient }\end{array}$ & $\underset{2}{2} \rightarrow$ & 20 & $\begin{array}{l}8=3 \\
0-8\end{array}$ & 5 \\
\hline $\begin{array}{l}\text { Max value of } \\
\text { the coefficient }\end{array}$ & 2 & 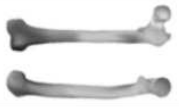 & $8=0$ & 8 \\
\hline $\begin{array}{l}\text { Number of the } \\
\text { mode }\end{array}$ & Mod 5 & Mod 6 & Mod 7 & Mod 8 \\
\hline $\begin{array}{l}\text { Min value of } \\
\text { the coefficient }\end{array}$ & $\sum_{-\infty}$ & $\begin{array}{l}8 \\
\infty\end{array}$ & $\begin{array}{l}\mathrm{E} \\
\mathrm{B}\end{array}$ & $\begin{array}{l}0.9 \\
0=-\infty\end{array}$ \\
\hline $\begin{array}{l}\text { Max value of } \\
\text { the coefficient }\end{array}$ & $\rightarrow$ & $\begin{array}{l}\varepsilon \\
2\end{array}$ & $\infty$ & 2 \\
\hline
\end{tabular}

Fig. 28. 3D visualization of mean value and first eight modes of femur bones (for each bone the anterior and posterior view is shown). 


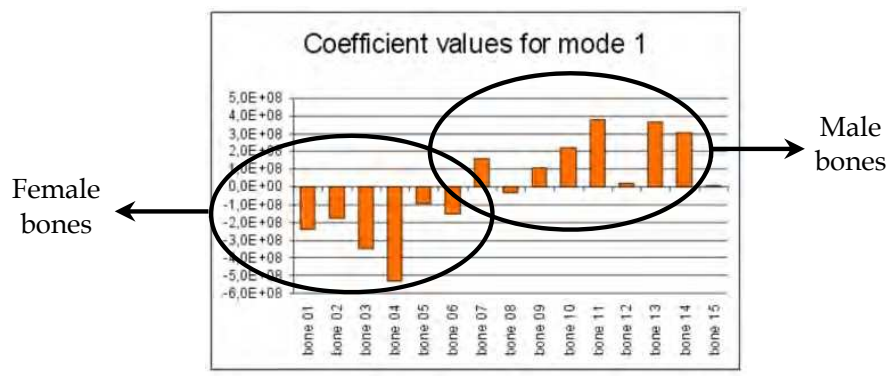

Fig. 29. Correlation between coefficient value of first mode and gender: the negative value "_" is correlated to female bone, positive value "+" is associated with male bones.

Other interesting feature of coefficients is individual set of coefficients values for each bone. This aspect is similar to "fingers prints". Because each bone have another geometry also they have individual set of coefficient values. On Figure 30 is presented graphs of coefficient values and visualization of the geometry for three different bones.
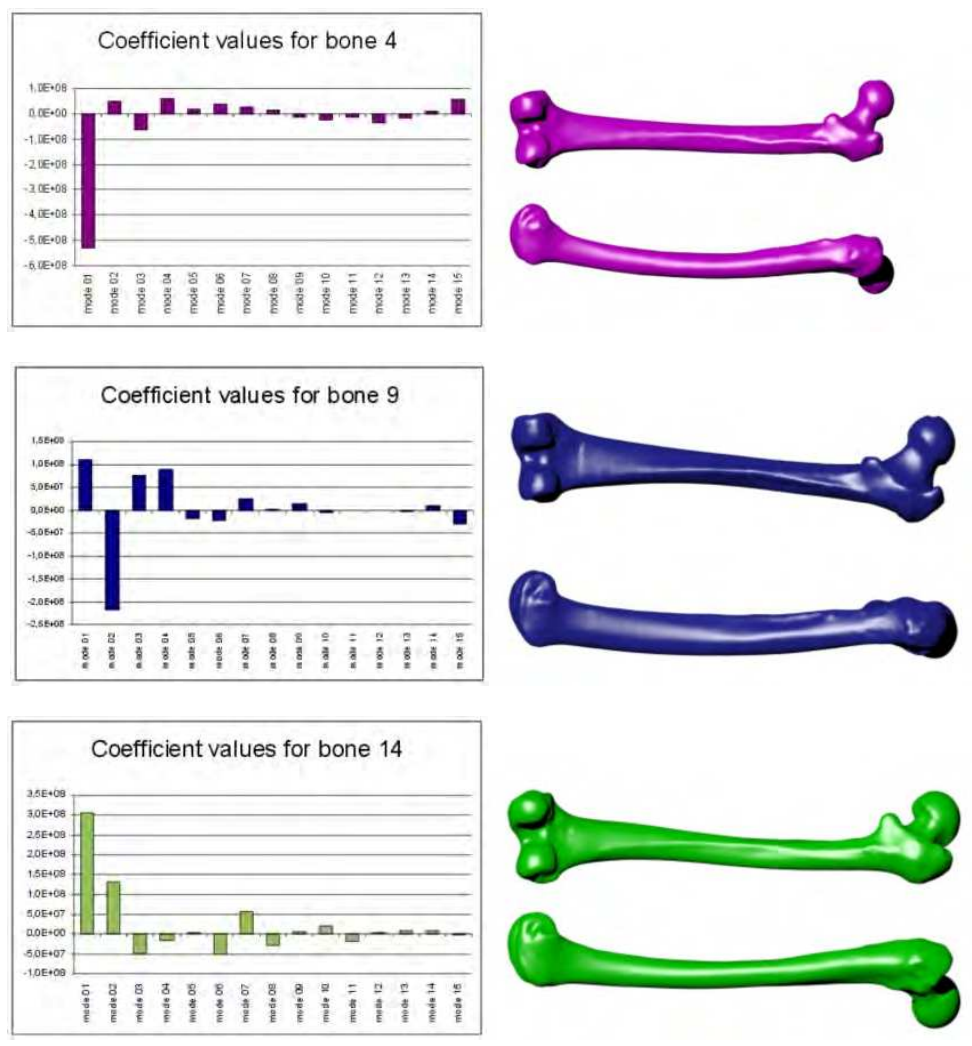

Fig. 30. Correlation between coefficient value and geometry of the bone for three different femur bones (all pictures of bones are made in the same scale). 


\subsection{Reconstruction method of 3D geometry of the bones basis on RTG images and empirical modes database}

Another advantage of property of real 3D anthropometric database is possibility of using this information as the "knowledge base". Knowledge base is necessary for the reconstruction of 3D geometry, basing on few RTG images (e.g. two or three ,depends from complication of the object shape). Method developed by authors [Rychlik M., 2004, Rychlik M. at al., 2005] can be used instead of CT images processing. The result of the reconstruction is 3D virtual model which can be used for develop individual prosthesis. The high accuracy (comparison with $\mathrm{CT}$ and contact 3D scanners) and low costs, making this method more common.

\subsubsection{Alghorithm of the reconstruction method}

The Algorithm of the method is following (Fig. 31.). Searched three-dimensional object are represented by the set of RTG images (minimum two images from different directions). These RTG images are compared with DRR - Digitally Reconstructed Radiographs [Milickovic N. at al. 2000, Russacof D. B. 2003] from database.

Data base includes DRR images and set of the modes and coefficients. Modes and coefficients are received from Principal Component Analysis. After comparison of images, the most similar objects from database are selected. In the next step we manipulate the coefficients of modes as long as the minimization of the mean square deviation of the images RTG and DRR is accomplished. Finally we receive reconstructed 3D model in CAD system.

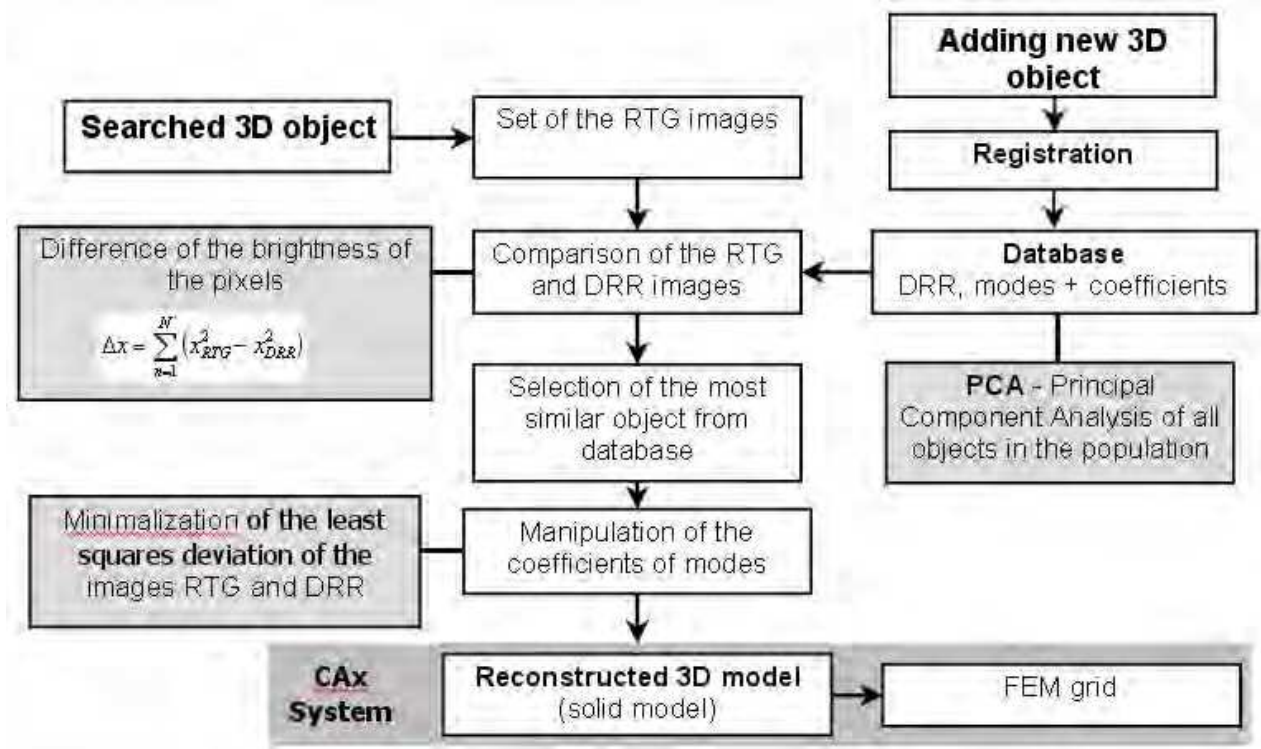

Fig. 31. Algorithm of the reconstruction method. 


\subsubsection{Input data - Artifical database}

The database used in experiment contains 99 lumbar vertebra's (Fig. 32.). Each vertebra has different geometry, and is described by FEM grid with the same structure (616 nodes, 2000 elements).
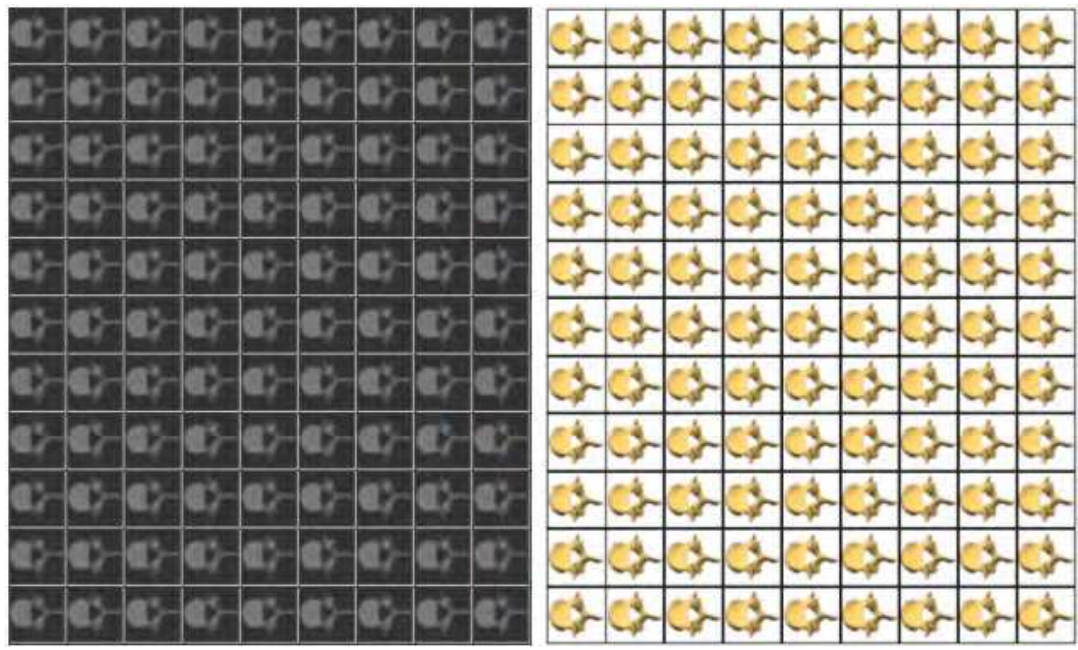

Fig. 32. Data base: DDR images (left side), 3D CAD models (right side).

\subsubsection{Results of 3D PCA analysis - Vertebra bones}

For this database the Principal Component Analysis was done. The result of this operation is the mean object, 11 modes and coefficients.

The first five modes include $96 \%$ of information about reconstructed geometry (Table 5.). Modes: twelfth and others contain very low information (this is only a numerical noise) and they aren't used for further reconstruction.

Modes describe the features of the vertebras (Fig. 33.). The first and fourth mode describe the deformation of the vertebral body, the second, third and seventh mode represent the deformation of the spinosus process. Other modes describe the deformation of the transverse process.

To verify this method the new vertebra has been made. It is deformed by three features (spinous process, vertebral body, transverse process) but has completely new values (there is no similar shape in data base).

In the next step we compare the DRR images of the searched and created vertebra and manipulate of the coefficients of modes. To compute the values of coefficients for all modes Jacobi criterion was used.

The final result of this experiment is the solid CAD model (Fig. 34.) and FEM grid. 


\begin{tabular}{ccc}
\hline $\begin{array}{c}\text { Number of } \\
\text { the mode }\end{array}$ & $\begin{array}{c}\text { Participation of } \\
\text { the mode [\%] }\end{array}$ & $\begin{array}{c}\text { Total participation } \\
\text { of the modes [\%] }\end{array}$ \\
\hline 1 & 60,7418270 & 60,7418270 \\
2 & 18,7637347 & 79,5055617 \\
3 & 8,0275130 & 87,5330747 \\
4 & 7,5452177 & 95,0782924 \\
5 & 1,1870943 & 96,2653867 \\
6 & 0,9569025 & 97,2222892 \\
7 & 0,6885226 & 97,9108118 \\
8 & 0,5571059 & 98,4679177 \\
9 & 0,5541704 & 99,0220881 \\
10 & 0,5054029 & 99,5274910 \\
11 & 0,4725086 & 99,9999996 \\
12 & 0,0000002 & 99,9999998 \\
13 & 0,0000001 & 99,9999999 \\
\hline
\end{tabular}

Table 5. Participation of the modes in reconstruction.

\begin{tabular}{|c|c|c|c|c|c|c|}
\hline $\begin{array}{l}\text { Number of the } \\
\text { mode }\end{array}$ & Mod 1 & Mod 2 & Mod 3 & Mod 4 & Mod 5 & Mod 6 \\
\hline \multicolumn{7}{|l|}{$\begin{array}{l}\text { Max value of } \\
\text { the coefficient }\end{array}$} \\
\hline \multicolumn{7}{|l|}{$\begin{array}{l}\text { Min value of } \\
\text { the coefficient }\end{array}$} \\
\hline $\begin{array}{l}\text { Number of the } \\
\text { mode }\end{array}$ & Mod 6 & Mod 7 & Mod 8 & Mod 9 & Mod 10 & Mod 11 \\
\hline \multicolumn{7}{|l|}{$\begin{array}{l}\text { Max value of } \\
\text { the coefficient }\end{array}$} \\
\hline $\begin{array}{l}\text { Min value of } \\
\text { the coefficient }\end{array}$ & & & & & & \\
\hline
\end{tabular}

Fig. 33. Three dimensional visualization of 11 modes.

To verify the quality of the reconstruction, the anthropometric measurements [Berry J. L., 1987] of the reconstructed vertebra was done. Average inaccuracy of the reconstruction is about $0,25 \mathrm{~mm}(\sim 1 \%)$. Volume differences between searched and reconstructed vertebra is about $0,11 \%$ and surface difference is $0,95 \%$. 

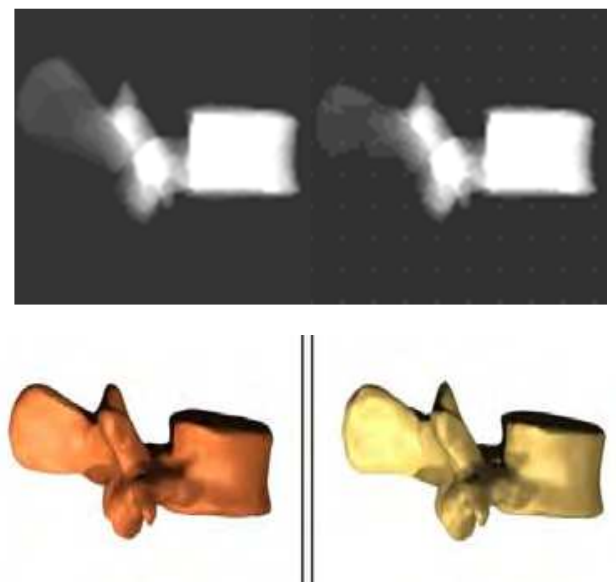

Fig. 34. Correlated images (from left): RTG image of searched object, DRR image of created (deformed) model, image of CAD solid model searched vertebra (left side) \& reconstructed vertebra (right side).

\section{Conclusions}

Many modern methods and techniques known from classic mechanics and engineering solutions, they are applicable in many other disciplines of knowledge. One of such new "beneficent" is biological branch of knowledge. In presented research authors concentrate attention on present several applications of three-dimensional version of Principal Component Analysis in biological cases. However to describe geometry of $3 \mathrm{D}$ objects the numerous modal methods can be used; only empirical modes gives an optimal statistical database. Empirical modes, represent features of the objects composed in database. Characters of features are dependent from frequent occurrence in population.

The first presented application of 3D PCA is biometrics. Many security systems need ID key, which will be fast and guarantees high level of protection. The 3D PCA make possible using 3D faces as the access code (Faceprint). Each face has individual set of coefficient values unique face code. That information (code) can be easily recorded onto electronic ID card similar to 2D biometric data's. Presented method as the source of data-input, apply cheap non-contact measurement method and full 3D face information - instead of "control" points set (few nodal points in "golden triangle" area). The 3D geometry of the face (3D faceprint) is more complicated than "flat" image and by this way more proof onto fake, than 2D face recognition systems. Other advantage of this method is automatically receiving unauthorized attempt (face of unauthorized persons) without require specialist decipher. For results interpretation of verification procedure, any special equipment or knowledge is not necessary. Results of verification can be understanding for everybody (without special training) and used immediately as the image or 3D model, for further analysis, e.g.: in police departments or other 3D modeling. 
Presented method was tested onto very specific group of data - faces of twins. Results of this analysis, was confirmed that even for very similar monozygotic twins faces (which can be very difficult for distinguish by human visual perception) the numerical ID - code of each face, was different and individual. Trends curves of coefficient values of modes have general the same trajectory but in local comparison still they are different. Them bigger difference between faces them larger distance between trend curves. For comparison of two faces of different twins the trend curves are completely different analogical like for two different persons. The basic input information for PCA, not necessary must be limited only to geometry of the object, but can be enlarged by additional information's (e.g. thermal images) into multi-dimensional database. In presented paper the $2 \mathrm{D}$ infrared images of faces was added to the basic database. Also this data can be coded and used as a thermal faceprint. Big disadvantage of thermal images of face surface is strong influence of environment temperature. As the more stabile and independent from temperature of atmosphere, the system based on thermal images of face veins is suggested.

Second presented application of 3D PCA analysis is anthropometrics. 3D PCA make possible extraction of mean shape and geometrical features of biological objects set. Mean shape characterize all 3D body (not only few 2D dimensions, like it is, in traditional anthropometric database). Features are describing principal deformations of analyzed group of objects (bones). This method can be used to create of full three dimensional anthropometric database of the skeleton system. One of advantages of that three dimensional anthropometric database, is possibility of measured any necessary dimensions on the surface of the mean bone. Mean shape and geometrical features (knowledge about shape and trends of deformations) can be used for developing new, more useful types of prosthesis. Such 3D anthropometrics analysis also can be important in anthropology, gives us information about the changes that appear in the human skeletal structure in different populations or ages.

As a third application the method of reconstruction of three-dimensional shape of biological object was presented. The reconstructed geometry is compatible with CAD systems. Reconstruction algorithm, developed by authors, is based on the few 2D RTG images and knowledge about object geometry recorded in empirical database. Presented method use full volume information from RTG images and can be used not only for reconstruction of the biological objects.

Accuracy of the method is higher than reconstructions based on CT-imagining and comparable with 3D scanners (about $0,3 \mathrm{~mm}$ ). Important characteristic of presented method is the automation of searching of the solution (elimination of landmarks) and that this method is non-invasive and non-destructive.

\section{Future directions of research}

Based onthe resultsof analysis presented above, in the following chapter authors propose further directions of researches. This work would have to expand possibilities of existing 3DPCA and to examine other methods of statistical analysis, such as: ICA-Independent Component Analysis. 
For application of PCA in 3D biometric systems the unified system of data recording (included areas of measurements) must develop. Furthermore, the optimal data acquisition process should be clearly defined for obtain highest accuracy level of measurement during the shortest time and the maximum simplification of the measuring equipment. An important element that should be subjected to further testing is the issue of facial expressions of the subjects. This aspect strongly influence on the obtained results. Also study of the influence of other objects such as glasses, beard, hat, etc., on the results should be examined. Considering the three-dimensional biometric system can be interesting to increase the number of dimensions included in the analysis of the PCA. Such additional information may be infrared images. However in this case, user must remember about strong impact of the ambient temperature on the obtained results. One solution to this problem may be the use of thermal images containing the facial structure of blood vessels.

Development of the PCA method in the anthropometric application should include the development of precise and effective tool of registration process. This procedure should include the specific areas of individual bones and the anatomical elements of the human body. Very interesting results can provide a PCA analysis of other human organs. Also basic research on the structure of the external shape of human body would have produced very valuable results for the ergonomic environment.

Another direction of development of the above considerations is the method of threedimensional reconstruction of the geometry of the object. Described method basis on the two-dimensional X-ray images and the 3D database generated by PCA analysis. For correct reconstruction is necessary to make set of measurements and analysis, contains: geometry measurements, PCA analysis and verification of reconstruction process performed on the of RTG images. Develop a comprehensive; coherent system for the reconstruction of geometry could allow the significantly spread the use of computer models in everyday medical practice.

\section{Acknowledgment}

The part of this work was supported under research grant no: N518 496039 from the Polish Ministry of Science 2010-2012.

\section{References}

Akhloufi, M, Bendada A., 2008,Infrared Face Recognition Using Distance Transforms, World Academy of Science, Engineering and Technology Nr 40, pp. 160-163.

Anderson, R. J., (2008), Security Engineering: A Guide to Building Dependable Distributed Systems, Wiley Publishing Inc., Indiana, USA ISBN-13: 9780470068526.

Anil K. J., Prabhakar S., Pankanti S., (2002), On the Similarity of identical twin fingerprints, The Journal of Patter Recognition Society Nr 35, pp. 2653 -2663.

Benameur S., Mignote M., Parent S., Labelle H., Skalli W., De Gusie J., (December 2001), 3D Biplanar Reconstruction of Scoliotic Vertebrae Using Statistical Models. 20th IEEE International Conference on Computer Vision and Pattern Recognition, CVPR'01, volume II, pp. 577-582. 
Berry J. L., Moran J. M., Berg W. S.,Steffee A. D., (1987), A morphometric study of human lumbar and selected thoracic vertebrae,Spine, Volume 12, Number 4.

Bro-Nielsen, M., Gramkow, C., (1996), Fast fluid registration of medical images, In: Proc. Visualization in Biomedical Computing (VBC'96), Springer Lecture Notes in Computer Science, vol. 1131, Hamburg

Holmes P., Lumley J. L., Berkooz G., (1996),Turbulence, coherent, structures dynamical systems and symmetry, Cambridge University Press.

Holmes, P., Lumley, J., L., Berkooz, G., (1998), Turbulence, Coherent Structures, Dynamical Systems and Symmetry, Cambridge University Press, Cambridge, New Edition.

Jain A.K., Ross A. and Pankanti S., (1999) , A Prototype Hand Geometry-based Verification System, Proceedings of 2nd International Conference on Audio- and Video-based Biometric Person Authentication (AVBPA), (Washington D.C.), pp.166-171.

Jantz, R.L. and P.H. Moore-Jansen, (1988), A Data Base for Forensic Anthropology: Structure, Content and Analysis, Report of Investigations no 47. Knoxville, TN: University of Tennessee.

Mainguet J-F. Biometrics, (2004), URL: http:// pagesperso-orange.fr/fingerchip/biometrics/biometrics.htm

Milickovic N., Baltas D., Giannouli S., Lahanas M., Zamboglou N., (2000)CT imaging based digitally reconstructed radiographs and its application in brachytherapy. Phys. Med. Biol. 45 , pp. $2787-2800$.

Moore-Jansen, P.M., S.D. Ousley, and R.L. Jantz, (1994), Data Collection Procedures for Forensic Skeletal Material. Report of Investigations no 48. Knoxville, TN: University of Tennessee.

Morzynski M., Afanasiev K., Thiele F., (1999). Solution of the eigenvalue problems resulting from global non-parallel flow stability analysis, Computer Methods Applied in Mechanical Engineering.

Prokoski. F., (2000) History, Current Status, and Future of Infrared Identification, IEEE Workshop on Computer Vision behind the Visible Spectrum: Methods and Applications (CVBVS 2000), pp 5-14.

Russacof D. B., Rohlfing T., Rueckert D., Shahidi R., Kim D., (2003), Maurer Calvin R. Fast calculation of digitally reconstructed radiographs using light fields, Medical Imagining 2003, Image Processing, Proceedings SPIE Vol. 5032.

Rychlik M., (2004), Original title In Polish: Metoda odtwarzania złożonych ksztattów przestrzennych dla systemów CAD, przy zredukowanej ilości danych pomiarowych, $\mathrm{PhD}$ work, Poznań University of Technology, Poznan Poland.

Rychlik M., Morzyński M., Stankiewicz W., (2005), Applications of CFD and PCA methods for geometry reconstruction of $3 D$ objects, Proceedings of the $10^{\text {th. }}$ International Conference Mathematical Modeling and Analysis, Vilnius, pp 123-128. ISBN 998605-924-0.

Sanchez-Reillo R., Sanchez-Avila and Gonzales-Marcos A., (2000), Biometric Identification Through Hand Geometry Measurements, IEEE Transactions on Pattern Analysis and Machine Intelligence.

Schneider W., (2007), Report of the Defense Science Board Task Force on Defense Biometrics, Department of Defense United States of America, Washington D.C. 
Syn, M., H-M., Prager, R., W., (1994), Mesh models for three-dimensional ultrasound imagining, CUED/F-INFENG/TR 210, Cambridge University Engineering Department, Cambridge.

Vitek C., (2005), A comparison in osteological measurements of two populations from East Tennessee, University of Tennessee at Chattanooga.

Vranic, D., Saupe, D., (2002), Description of 3D-shape using a complex function of the sphere, Department of Computer and Information Science, University of Konstanz.

Xiaoguang Lu, (2006), 3D Face Recognition across Pose and Expression, PhD thesis, Michigan State University 


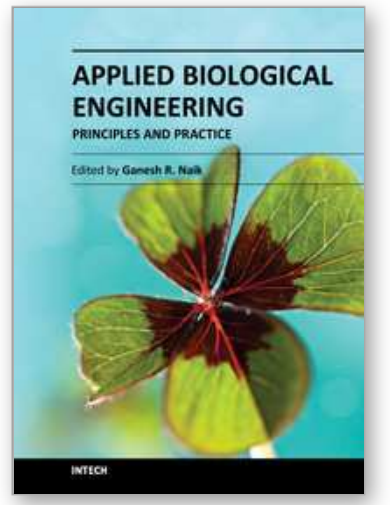

\author{
Applied Biological Engineering - Principles and Practice \\ Edited by Dr. Ganesh R. Naik
}

ISBN 978-953-51-0412-4

Hard cover, 662 pages

Publisher InTech

Published online 23, March, 2012

Published in print edition March, 2012

Biological engineering is a field of engineering in which the emphasis is on life and life-sustaining systems. Biological engineering is an emerging discipline that encompasses engineering theory and practice connected to and derived from the science of biology. The most important trend in biological engineering is the dynamic range of scales at which biotechnology is now able to integrate with biological processes. An explosion in micro/nanoscale technology is allowing the manufacture of nanoparticles for drug delivery into cells, miniaturized implantable microsensors for medical diagnostics, and micro-engineered robots for on-board tissue repairs. This book aims to provide an updated overview of the recent developments in biological engineering from diverse aspects and various applications in clinical and experimental research.

\title{
How to reference
}

In order to correctly reference this scholarly work, feel free to copy and paste the following:

Michal Rychlik and Witold Stankiewicz (2012). Extraction of 3D Geometrical Features of Biological Objects with 3D PCA Analysis and Applications of Results, Applied Biological Engineering - Principles and Practice, Dr. Ganesh R. Naik (Ed.), ISBN: 978-953-51-0412-4, InTech, Available from:

http://www.intechopen.com/books/applied-biological-engineering-principles-and-practice/extraction-of-3dgeometrical-features-of-biological-objects-with-3d-pca-analysis-and-results-applica

\section{INTECH}

open science | open minds

\author{
InTech Europe \\ University Campus STeP Ri \\ Slavka Krautzeka 83/A \\ 51000 Rijeka, Croatia \\ Phone: +385 (51) 770447 \\ Fax: +385 (51) 686166 \\ www.intechopen.com
}

\author{
InTech China \\ Unit 405, Office Block, Hotel Equatorial Shanghai \\ No.65, Yan An Road (West), Shanghai, 200040, China \\ 中国上海市延安西路65号上海国际贵都大饭店办公楼 405 单元 \\ Phone: +86-21-62489820 \\ Fax: +86-21-62489821
}


(C) 2012 The Author(s). Licensee IntechOpen. This is an open access article distributed under the terms of the Creative Commons Attribution 3.0 License, which permits unrestricted use, distribution, and reproduction in any medium, provided the original work is properly cited. 